\title{
Design Optimisation of Lower-Bound Buckling Capacities for FRP-Laminated Cylindrical Shells
}

\author{
Hongtao Wang and James G. A. Croll \\ Department of Civil and Environmental Engineering, University College London, Gower Street, London WC1E 6BT, UK \\ Correspondence should be addressed to James G. A. Croll, j.croll@ucl.ac.uk
}

Received 25 October 2011; Accepted 1 December 2011

Academic Editor: W.-H. Steeb

Copyright ( $) 2012$ H. Wang and J. G. A. Croll. This is an open access article distributed under the Creative Commons Attribution License, which permits unrestricted use, distribution, and reproduction in any medium, provided the original work is properly cited.

\begin{abstract}
The imperfection sensitive buckling loads of fibre reinforced polymeric (FRP) composite cylindrical shells under axial compression can be optimised with respect to many material and geometric parameters. Current approaches, using mathematical algorithms to optimise the linearised classical critical loads with respect to many design variables, generally ignore the potential reductions in elastic load carrying capacities that result from the severe sensitivities of buckling loads to the effects of initial imperfections. This paper applies a lower-bound design philosophy called the reduced stiffness method (RSM) to the optimisation design of FRP shell buckling. A physical optimisation in terms of parametric studies is carried out for simply supported, 6-ply symmetric, glassepoxy circular cylindrical shells under uniform axial load. It is shown that under the guidance of RSM, safe lower-bound buckling loads can be enhanced greatly by choosing appropriate combinations of design parameters. It is demonstrated how this approach encourages the delineation of those components of the shell's membrane and bending stiffness that are important and those that are unimportant within each of the prospective buckling modes. On this basis, it is argued that the RSM provides not only a safe but also a more rational strategy for better design decision making.
\end{abstract}

\section{Introduction}

Due to their high strength-to-stiffness and strength-toweight ratios, fibre-reinforced-polymeric- (FRP-) laminated shells are widely used in the weight sensitive industries such as aerospace, automobile, and offshore engineering. For thin FRP-laminated shells, the typically low elastic stiffness-tostrength ratios result in the elastic buckling playing a greater role in the design process compared with equivalent metallic structures. Relative to conventional metallic shells, the buckling capacities of FRP laminated shells will depend upon a much larger number of additional design variables, such as fibre distribution and orientation, lamina stacking sequence and thickness, and material selections. Identifying optimum design choices is consequently a more complex problem than for metallic shells.

Current approaches [1-5] to the problem share a methodology based upon mathematical optimisation algorithms seeking the maximum linear classical critical loads of perfect
FRP-laminated shells with respect to many design parameters. These approaches have inherent defects. Firstly they heavily rely on immense mathematical and computational efforts. Secondly they usually leave basic understanding of the mechanics of buckling implicit and consequently provide little direct assistance to the designer. Thirdly, and more importantly, real shells always have initial geometric imperfections. These imperfections play a defining role as to the reductions of elastic buckling capacities from the unsafe upper bound provided by the classical critical loads. Different design parameters and different buckling modes will exhibit very different levels of imperfection-related knock-down to safe, lower-bound, buckling loads. Therefore, optimising the unsafe buckling loads of the perfect laminated composite shells has no direct or obvious relevance to the identification of the optimal safe buckling loads of the corresponding imperfect shells. Assuming that imperfect laminated composite shells have the same optimal laminate configurations for buckling resistance as perfect ones can be 


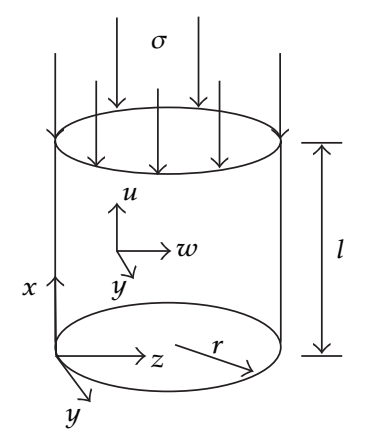

FIgURE 1: Convention for shell geometry and coordinate.

quite erroneous. Hence past predictions of optimal laminate configurations for perfect shells may not prove optimal when imperfections are taken into account.

Although some researchers have tried to assess the imperfection sensitive buckling loads of the optimised composite shells by using Koiter's initial postbuckling theory, their predictions are usually higher than the experimental data, for example [3], and assume that the most severe imperfection sensitivity will always occur in the mode for which the classical theory predicts a minimum. Also, it is believed $[6,7]$ that optimal classical critical loads are generally associated with increased imperfection sensitivity. The presence of initial imperfections may make these apparent optimal configurations for perfect shells less favorable than other designs as a result of modes that display lower imperfection sensitive buckling loads. Therefore, these approaches are far away from serving as rational optimisation design methods.

A rational design method of optimising buckling capacities of laminated composite shells should take account of the effects of initial imperfections at the outset. Furthermore, it should be able to give safe predictions of the imperfection sensitive buckling loads. But initial imperfections are stochastic variables. Before shells are built, no information about the details of initial imperfections can be obtained. On the other hand, once shells are built, there is no need to carry out optimisation. Without the information of initial imperfections and complete knowledge of the effects of all forms of initial imperfections on buckling resistance of laminated composite shells, it may reasonably be asked whether a rational design philosophy to optimise the buckling capacities of composite shells is possible. The answer we believe is positive.

Since it is impractical to obtain concrete information on initial imperfections before a shell is built and because the effects of various initial imperfections are quite different from one another, one possible way to tackle this dilemma is to predict the worst effects of initial imperfections on the buckling capacities of composite shells. The reduced stiffness method (RSM) is such a lower-bound design philosophy that is able to predict the worst possible effects of initial imperfections. It is based on the physical argument that the reductions in the buckling loads of shells result from the loss of initial stabilising membrane energy in the postbuckling regime, due to mode couplings catalysed by the presence of imperfections. By eliminating these membrane energies, the classical critical analysis allows a lower bound to the imperfection sensitive buckling loads of shells to be obtained. The RSM has predicted safe lower bounds for a range of isotropic and stiffened shells [8]. Since this method also encourages the delineation of those components of the shell's membrane and bending stiffnesses that are important and those that are unimportant within each of the prospective buckling modes, it also provides a valuable tool for better design decision making. This paper provides a brief outline of the RSM applied to the prediction of safe buckling capacities of laminated composite shells. It attempts to demonstrate how the optimisation based upon the safe, lower-bound, predictions of the RSM leads to designs that could be very different from those based upon unsafe, upperbound predictions.

\section{Analytical Formulation}

The geometry and coordinate system of an FRP-laminated circular cylindrical shell are shown in Figure 1 with thickness, $t$, length, $l$, and radius, $r$. The shell is simply supported and subject to uniform axial stress $\sigma$.

2.1. Kinematic Relations. The Donnell approximations of the linear incremental strain-displacement relations at the critical state are

$$
\begin{array}{cc}
\varepsilon_{x}^{\prime}=\frac{\partial u}{\partial x}, & \chi_{x}^{\prime}=-\frac{\partial^{2} w}{\partial x^{2}}, \\
\varepsilon_{\theta}^{\prime}=\frac{1}{r}\left(\frac{\partial v}{\partial \theta}-w\right), & \chi_{\theta}^{\prime}=-\frac{1}{r^{2}} \frac{\partial^{2} w}{\partial \theta^{2}}, \\
\varepsilon_{x \theta}^{\prime}=\frac{\partial v}{\partial x}+\frac{1}{r} \frac{\partial u}{\partial \theta}, & \chi_{x \theta}^{\prime}=-\frac{1}{r} \frac{\partial^{2} w}{\partial x \partial \theta},
\end{array}
$$

and the non-linear incremental components

$$
\begin{aligned}
& \varepsilon_{x}^{\prime \prime}=\frac{1}{2}\left(\frac{\partial w}{\partial x}\right)^{2}, \\
& \varepsilon_{\theta}^{\prime \prime}=\frac{1}{2 r^{2}}\left(\frac{\partial w}{\partial \theta}\right)^{2} .
\end{aligned}
$$




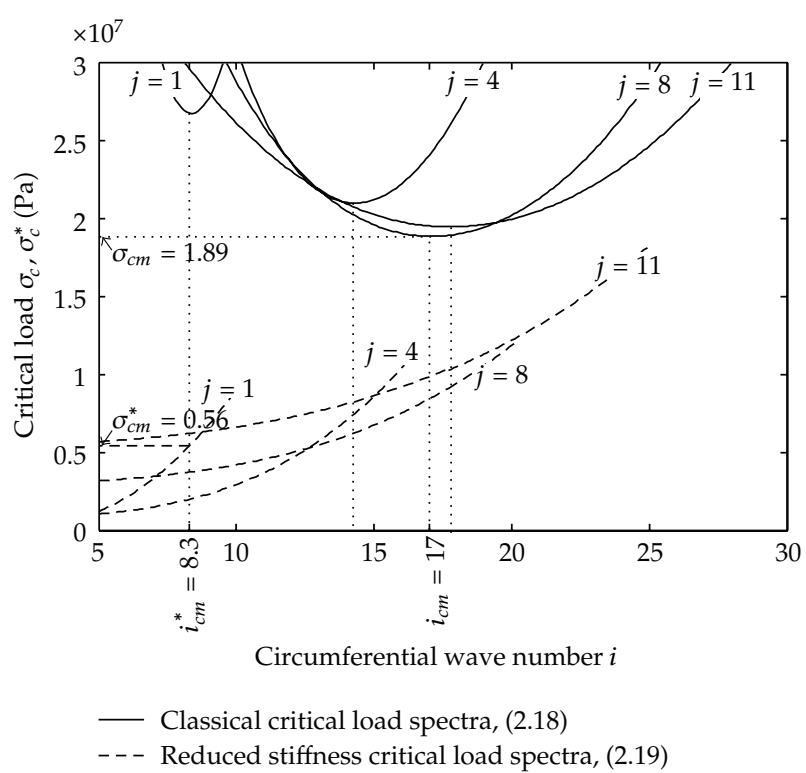

FIgURE 2: The classical critical load and reduced stiffness critical load analyses for the shell $\left[0^{\circ}\right]_{6}$.

Here $u, v$, and $w$ denote incremental displacements in the axial, circumferential, and radial directions from the critical state; $\left(\varepsilon_{x}, \varepsilon_{\theta}, \varepsilon_{x \theta}\right)$ are the incremental membrane strains; $\left(\chi_{x}, \chi_{\theta}, \chi_{x}\right)$ are the incremental bending strains. A single dash superscript denotes the linear components, and a double dash the quadratic components.

2.2. Constitutive Relations. Based on the classical lamination theory, the constitutive relations corresponding to the linear incremental strain components are

$$
\begin{aligned}
& \left\{\begin{array}{c}
n_{x}^{\prime} \\
n_{\theta}^{\prime} \\
n_{x \theta}^{\prime}
\end{array}\right\}=\left[\begin{array}{lll}
A_{11} & A_{12} & A_{16} \\
A_{12} & A_{22} & A_{26} \\
A_{16} & A_{26} & A_{66}
\end{array}\right]\left\{\begin{array}{c}
\varepsilon_{x}^{\prime} \\
\varepsilon_{\theta}^{\prime} \\
\varepsilon_{x \theta}^{\prime}
\end{array}\right\}+\left[\begin{array}{lll}
B_{11} & B_{12} & B_{16} \\
B_{12} & B_{22} & B_{26} \\
B_{16} & B_{26} & B_{66}
\end{array}\right]\left\{\begin{array}{c}
\chi_{x}^{\prime} \\
\chi_{\theta}^{\prime} \\
2 \chi_{x \theta}^{\prime}
\end{array}\right\}, \\
& \left\{\begin{array}{l}
m_{x}^{\prime} \\
m_{\theta}^{\prime} \\
m_{x \theta}^{\prime}
\end{array}\right\}=\left[\begin{array}{lll}
B_{11} & B_{12} & B_{16} \\
B_{12} & B_{22} & B_{26} \\
B_{16} & B_{26} & B_{66}
\end{array}\right]\left\{\begin{array}{c}
\varepsilon_{x}^{\prime} \\
\varepsilon_{\theta}^{\prime} \\
\varepsilon_{x \theta}^{\prime}
\end{array}\right\}+\left[\begin{array}{lll}
D_{11} & D_{12} & D_{16} \\
D_{12} & D_{22} & D_{26} \\
D_{16} & D_{26} & D_{66}
\end{array}\right]\left\{\begin{array}{c}
\chi_{x}^{\prime} \\
\chi_{\theta}^{\prime} \\
2 \chi_{x \theta}^{\prime}
\end{array}\right\},
\end{aligned}
$$

and those corresponding to the nonlinear incremental strain components are

$$
\left\{\begin{array}{l}
n_{x}^{\prime \prime} \\
n_{\theta}^{\prime \prime}
\end{array}\right\}=\left[\begin{array}{ll}
A_{11} & A_{12} \\
A_{12} & A_{22}
\end{array}\right]\left\{\begin{array}{c}
\varepsilon_{x}^{\prime \prime} \\
\varepsilon_{\theta}^{\prime \prime}
\end{array}\right\} .
$$

Following the usual convention $\left(n_{x}, n_{\theta}, n_{x \theta}\right)$ are the stress resultants; $\left(m_{x}, m_{\theta}, m_{x \theta}\right)$ are the incremental moment resultants.

$A_{i j}, B_{i j}$, and $D_{i j}(i, j=1,2,6)$ are, respectively, the membrane, bending-membrane coupling, and bending stiffnesses of a laminate and have the following definitions:

$$
\left(A_{i j}, B_{i j}, D_{i j}\right)=\int_{-t / 2}^{t / 2} \bar{Q}_{i j}\left(1, z, z^{2}\right) d z \quad(i, j=1,2,6) .
$$

Here $\bar{Q}_{i j}(i, j=1,2,6)$ are the transformed in-plane stiffnesses of a single ply and are defined as follows:

$$
\begin{aligned}
& \overline{\mathcal{Q}}_{11}=\mathcal{Q}_{11} \cos ^{4} \theta+2\left(\mathcal{Q}_{12}+2 \mathcal{Q}_{66}\right) \sin ^{2} \theta \cos ^{2} \theta+\mathcal{Q}_{22} \sin ^{4} \theta, \\
& \overline{\mathcal{Q}}_{12}=\left(\mathcal{Q}_{11}+\mathcal{Q}_{22}-4 \mathcal{Q}_{66}\right) \sin ^{2} \theta \cos ^{2} \theta+\mathcal{Q}_{12}\left(\sin ^{4} \theta+\cos ^{4} \theta\right) \text {, } \\
& \overline{\mathcal{Q}}_{22}=\mathcal{Q}_{11} \sin ^{4} \theta+2\left(\mathcal{Q}_{12}+2 \mathcal{Q}_{66}\right) \sin ^{2} \theta \cos ^{2} \theta+\mathcal{Q}_{22} \cos ^{4} \theta, \\
& \overline{\mathcal{Q}}_{16}=\left(\mathcal{Q}_{11}-\mathcal{Q}_{12}-2 \mathcal{Q}_{66}\right) \sin \theta \cos ^{3} \theta \\
& +\left(\mathcal{Q}_{12}-\mathcal{Q}_{22}+2 \mathcal{Q}_{66}\right) \sin ^{3} \theta \cos \theta \\
& \overline{\mathcal{Q}}_{26}=\left(\mathcal{Q}_{11}-\mathcal{Q}_{12}-2 \mathcal{Q}_{66}\right) \sin ^{3} \theta \cos \theta \\
& +\left(\mathcal{Q}_{12}-\mathcal{Q}_{22}+2 \mathcal{Q}_{66}\right) \sin \theta \cos ^{3} \theta \\
& \overline{\mathcal{Q}}_{66}=\left(\mathcal{Q}_{11}+\mathcal{Q}_{22}-2 \mathcal{Q}_{12}-2 \mathcal{Q}_{66}\right) \sin ^{2} \theta \cos ^{2} \theta \\
& +\mathcal{Q}_{66}\left(\sin ^{4} \theta+\cos ^{4} \theta\right)
\end{aligned}
$$

where $\theta$ is the angle of fibre orientation ranging from $-90^{\circ}$ to $90^{\circ}$ relative to the positive direction of the $x$-axis with the positive angle defined as rotating towards the positive direction of the $y$-axis; $Q_{i j}(i, j=1,2,6)$ are the in-plane stiffnesses of an orthotropic ply in the principle material directions and are defined as

$$
\begin{array}{ll}
\mathcal{Q}_{11}=\frac{E_{1}}{1-\mu_{12} \mu_{21}}, & \mathcal{Q}_{22}=\frac{E_{2}}{1-\mu_{12} \mu_{21}}, \\
\mathcal{Q}_{12}=\frac{\mu_{21} E_{1}}{1-\mu_{12} \mu_{21}}, & \mathcal{Q}_{66}=G_{12},
\end{array}
$$

with the reciprocal relation:

$$
\frac{\mu_{12}}{E_{1}}=\frac{\mu_{21}}{E_{2}}
$$

$E_{1}, E_{2}, \mu_{12}$, and $G_{12}$ are the apparent Young's modulus of a ply in the direction of the fibre, the apparent Young's modulus in the direction transverse to the fibres, the major Poisson's ratio, and the apparent in-plane shear modulus, respectively. To be able examine the parameters of fibre and matrix volume fractions $\left(V_{f}, V_{m}\right)$ and material properties, we use the Halpin-Tsai equations to determine the aforementioned four independent engineering constants and they are

$$
\begin{gathered}
E_{1} \cong E_{f} V_{f}+E_{m} V_{m}, \\
\mu_{12}=\mu_{f} V_{f}+\mu_{m} V_{m}, \\
\frac{M}{M_{m}}=\frac{1+\xi \eta V_{f}}{1-\eta V_{f}},
\end{gathered}
$$


where

$$
\eta=\frac{\left(M_{f} / M_{m}\right)-1}{\left(M_{f} / M_{m}\right)+\xi}
$$

in which $M$ is the composite material modulus $E_{2}$ or $G_{12} ; M_{f}$ is the corresponding fibre modulus $E_{f}, G_{f}$, or $\mu_{f} ; M_{m}$ is the corresponding matrix modulus $E_{m}, G_{m}$, or $\mu_{m}$; for $E_{2}, \xi=$ $2 ; G_{12}, \quad \xi=1+4 V_{f}^{10}$.

Detailed derivations of all the aforementioned stiffness parameters and the determination of engineering constants can be found in [9].

2.3. Fundamental State. For a circular cylindrical shell subject to uniform axial compression, the fundamental state prior to bifurcation point can be taken as axisymmetric membrane in the classical buckling analysis. Hence the fundamental stress resultants are

$$
\left(N_{x}^{F}, N_{\theta}^{F}, N_{x \theta}^{F}\right)=(-\sigma t, 0,0) .
$$

Here we assume that the effect of membrane-bending coupling stiffnesses $B_{i j}(i, j=1,2,6)$ can be neglected in the fundamental state, and that the extension-shear coupling stiffnesses $A_{16}$ and $A_{26}$ equal zero.

Using constitutive relations (3), the corresponding fundamental strain components are

$$
\left(E_{x}^{F}, E_{\theta}^{F}, E_{x \theta}^{F}\right)=\left(-\frac{A_{22} \sigma t}{A_{11} A_{22}-A_{12}^{2}}, \frac{A_{12} \sigma t}{A_{11} A_{22}-A_{12}^{2}}, 0\right) .
$$

2.4. Displacement Functions. For simply supported boundary conditions, the incremental displacements can be taken as harmonic functions:

$$
\begin{gathered}
u=u_{i j} \cos (i \theta) \cos \left(\frac{j \pi x}{l}\right), \\
v=v_{i j} \sin (i \theta) \sin \left(\frac{j \pi x}{l}\right), \\
w=w_{i j} \cos (i \theta) \sin \left(\frac{j \pi x}{l}\right),
\end{gathered}
$$

where $i$ is the circumferential full wave number and $j$ the axial half-wave number.

2.5. Classical Critical Load Analysis. In the classical critical analysis, the principle of stationary total potential energy gives a compact and systematic framework for interpreting buckling behaviour. Of present interest is the quadratic term of the total potential energy from which the condition of the stationarity results in the eigenvalue problem that yields the classical critical load spectra. The quadratic term of the total potential energy can be represented as

$$
V_{2}=U_{2 B}^{x}+U_{2 B}^{\theta}+U_{2 B}^{x \theta}+U_{2 M}^{x}+U_{2 M}^{\theta}+U_{2 M}^{x \theta}+V_{2 M}^{x}+V_{2 M}^{\theta},
$$

where $U_{2 b}^{x}=(1 / 2) \int_{0}^{1} \int_{0}^{2 \pi} m_{x}^{\prime} \chi_{x}^{\prime} r d \theta d x$ is the linear axial bending energy, $U_{2 B}^{\theta}=(1 / 2) \int_{0}^{1} \int_{0}^{2 \pi} m_{\theta}^{\prime} \chi_{\theta}^{\prime} r d \theta d x$ is the linear circumferential (hoop) bending energy, $U_{2 B}^{x \theta}=$ $(1 / 2) \int_{0}^{l} \int_{0}^{2 \pi} 2 m_{x \theta}^{\prime} \chi_{x \theta}^{\prime} r d \theta d x$ is the linear twist bending energy, $U_{2 M}^{x}=(1 / 2) \int_{0}^{l} \int_{0}^{2 \pi} n_{x}^{\prime} \varepsilon_{x}^{\prime} r d \theta d x$ is the linear axial membrane energy, $U_{2 M}^{\theta}=(1 / 2) \int_{0}^{l} \int_{0}^{2 \pi} n_{\theta}^{\prime} \varepsilon_{\theta}^{\prime} r d \theta d x$ is the linear circumferential membrane energy, $U_{2 M}^{x \theta}=(1 /$ 2) $\int_{0}^{l} \int_{0}^{2 \pi} n_{x \theta}^{\prime} \varepsilon_{x \theta}^{\prime} r d \theta d x$ is the linear shear membrane energy, $V_{2 M}^{x}=(1 / 2) \int_{0}^{l} \int_{0}^{2 \pi}\left(N_{x}^{F} \varepsilon_{x}^{\prime \prime}+n_{x}^{\prime \prime} E_{x}^{F}\right) r d \theta d x$ is the linearised axial membrane energy, and $V_{2 M}^{\theta}=$ (1/ 2) $\int_{0}^{l} \int_{0}^{2 \pi} n_{\theta}^{\prime \prime} E_{\theta}^{F} r d \theta d x$ is the linearised circumferential membrane energy.

Using (11), (12), and the expressions obtained from substituting (13) into (1)-(4), the condition of stationarity of the total potential energy with respect to arbitrary kinematically admissible displacements requires

$$
\frac{\partial V_{2}}{\partial u_{i j}}=0, \quad \frac{\partial V_{2}}{\partial v_{i j}}=0, \quad \frac{\partial V_{2}}{\partial w_{i j}}=0,
$$

which results in the linear eigenvalue problem as follows:

$$
\left[\begin{array}{lll}
C_{11} & C_{12} & C_{13} \\
C_{21} & C_{22} & C_{23} \\
C_{31} & C_{32} & C_{33}
\end{array}\right]\left\{\begin{array}{c}
u_{i j} \\
v_{i j} \\
w_{i j}
\end{array}\right\}+\sigma\left[\begin{array}{ccc}
0 & 0 & 0 \\
0 & 0 & 0 \\
0 & 0 & \varphi_{33}
\end{array}\right]\left\{\begin{array}{l}
u_{i j} \\
v_{i j} \\
w_{i j}
\end{array}\right\}=0,
$$

where

$$
\begin{aligned}
C_{11}= & A_{11} \lambda^{2}+A_{66} i^{2}, \\
C_{12}= & -\left(A_{12}+A_{66}\right) \lambda i, \\
C_{13}= & -\frac{\lambda}{r}\left[B_{11} \lambda^{2}+\left(B_{12}+2 B_{66}\right) i\right]+A_{12} \lambda, \\
C_{22}= & A_{22} i^{2}+A_{66} \lambda^{2}, \\
C_{23}= & \frac{i}{r}\left[B_{22} i^{2}+\left(B_{12}+2 B_{66}\right) \lambda^{2}\right]-A_{22} i, \\
C_{33}= & A_{22}+\frac{1}{r^{2}}\left[D_{11} \lambda^{4}+D_{22} i^{4}+2\left(D_{12}+2 D_{66}\right) \lambda^{2} i^{2}\right] \\
& -\frac{2}{r}\left(B_{12} \lambda^{2}+B_{22} i^{2}\right), \\
\varphi_{33}= & -\lambda^{2} t,
\end{aligned}
$$

in which $\lambda \equiv j \pi / L$.

The existence of nontrivial solutions of (16) requires the coefficient determinant to equal zero. Hence, we obtain the classical critical load spectra:

$\sigma_{c}=\frac{C_{22} C_{13}^{2}+C_{11} C_{23}^{2}+C_{33} C_{12}^{2}-C_{11} C_{22} C_{33}-2 C_{12} C_{23} C_{13}}{\left(C_{11} C_{22}-C_{12}^{2}\right) \varphi_{33}}$. 
2.6. Reduced Stiffness Analysis. Except for the nonlinear axial energy component $V_{2 M}^{x}$ that is negative, all the other energy components in (14) are positive definite. Except for $V_{2 M}^{x}$, this means that all the other energy components contribute to the shell's initial resistance to buckling. The reduced stiffness method is based on the physical argument that mode coupling, catalysed by geometric imperfections, results in the loss of initial stabilising membrane energy (see [8] for a recent summary). By eliminating $U_{2 M}$ and $V_{2 M}^{\theta}$ from (14) and applying the condition of stationarity to the reduced quadratic form of the total potential energy, we obtain the reduced stiffness critical load spectra:

$$
\sigma_{c}^{*}=\frac{2\left(A_{11} A_{22}-A_{12}^{2}\right)\left[-\left(B_{12} \lambda^{2}+B_{22} i^{2}\right) r+D_{11} \lambda^{4}+2\left(D_{12}+2 D_{66}\right) i^{2} \lambda^{2}+D_{22} i^{4}\right]}{\left[\left(2 A_{11} A_{22}-A_{12}^{2}\right) \lambda^{2}+A_{12} A_{22} i^{2}\right] r^{2} t} .
$$

2.7. Reduced Stiffness Optimisation Strategy. The neutral stability of the critical state requires the quadratic term of the total potential energy (14) to equal zero. So the classical critical loads can be expressed as

$$
\sigma_{\mathrm{c}}=\frac{U_{2 M}+U_{2 B}}{-\left(\partial V_{2 M}^{x} / \partial \sigma+\partial V_{2 M}^{\theta} / \partial \sigma\right)}
$$

The corresponding reduced stiffness critical loads are

$$
\sigma_{c}^{*}=\frac{U_{2 B}}{-\partial V_{2 M}^{x} / \partial \sigma}
$$

As will be shown in the following example, each choice of axial half-wave $j$ will result in a classical critical load spectrum from (20) that exhibits a minimum at some value of $i$. The lowest of these minima, occurring in a mode $(i, j)=\left(i_{c m}, j_{c m}\right)$, is what is usually referred to as the classical critical load, here denoted by $\sigma_{c m}$. For each choice of $j$, the reduced stiffness critical spectra of (21) will predict a value of $\sigma_{c}^{*}$ associated with the circumferential wave number $i$ corresponding to the lowest classical critical load. The lowest of these reduced stiffness critical loads $\sigma_{c m}^{*}$ will occur in a mode $(i, j)=\left(i_{c m}^{*}, j_{c m}^{*}\right)$ that could be different to the classical critical mode $\left(i_{c m}, j_{c m}\right)$. It is this least value $\sigma_{c m}^{*}$ that has been shown to represent a lower bound to imperfection sensitivity [10]. It is clear that the philosophy of the reduced stiffness optimisation is to increase the bending energy and decrease the destabilising effect of the nonlinear axial membrane energy to enhance the lower bound of the buckling capacities of shells. It will be shown that such a procedure will cause mode shifts that allow identification of an optimum combination of the many material and geometric parameters.

\section{Typical Case Study}

A 6-ply symmetric glass-epoxy circular cylindrical shell is taken as an example. The shell has the geometric and material properties as follows:

$$
\begin{aligned}
& \frac{l}{r}=2.048, \\
& \frac{r}{t}=405,
\end{aligned}
$$

$$
\begin{array}{ll}
E_{f}=72 \mathrm{GPa}, & \mu_{f}=0.22, \\
E_{m}=3.5 \mathrm{GPa}, & \mu_{m}=0.34 .
\end{array}
$$

3.1. Orthotropic Laminate Configurations. A shell $\left[0^{\circ}\right]_{6}$, which has all fibres orientated in the axial direction, with equal ply thickness is taken as the starting point. " 0 " " denotes the angle of fibre orientation and the subscript " 6 " denotes the number of ply. The fibre volume fraction is 0.5 for each ply. Figure 1 shows the classical critical load and the reduced stiffness critical load analyses for this shell. The classical critical load analysis predicts that the minimum critical load $\sigma_{c m}=18.9 \mathrm{MPa}$ occurs in the mode $\left(i_{c m}, j_{c m}\right)=(17,8)$ while the reduced stiffness analysis predicts that the lower bound $\sigma_{c m}^{*}=5.6 \mathrm{MPa}$ happens in the mode $\left(i_{c m}^{*}, j_{c m}^{*}\right)=(8.3,1)$ with nearly $70 \%$ reduction from the minimum classical critical load. To guide our choice as to how to rearrange lamina configuration to enhance the reduced stiffness critical load, we need to look at the different contributions from various energy terms in the classical critical modes.

Figure 3 shows that in mode $j=1$, for the case of $\left[0^{\circ}\right]_{6}$ the linear hoop bending energy is the major component within the total bending energy in the reduced stiffness critical mode. Since all membrane energies are eliminated from the classical critical load analysis, the most important strategy of the reduced stiffness optimisation is to ensure that the total bending energy in the reduced stiffness critical mode is as high as possible. An obvious way to improve the lower-bound buckling capacity of the shell is to enhance this important linear hoop bending energy. However, under current circumstances with fibre unidirectionally orientated in the axial direction and the total fibre volume fraction fixed, the objective of increasing the hoop bending energy is of necessity achieved by moving fibres from the axial direction to the hoop direction at the sacrifice of the axial membrane stiffness. From Figure 3, it can be seen that the linear axial membrane energy is the major component constituting the total linear membrane energy. Any decrease of the linear axial membrane energy is very likely to result in the decrease of the total linear membrane energy, which has the effect of lengthening the circumferential wavelength. That is to say that the classical critical mode $i_{c m}$ would shift towards the left in Figure 3, resulting in a reduction of the linear circumferential bending energy. The reduction in axial 


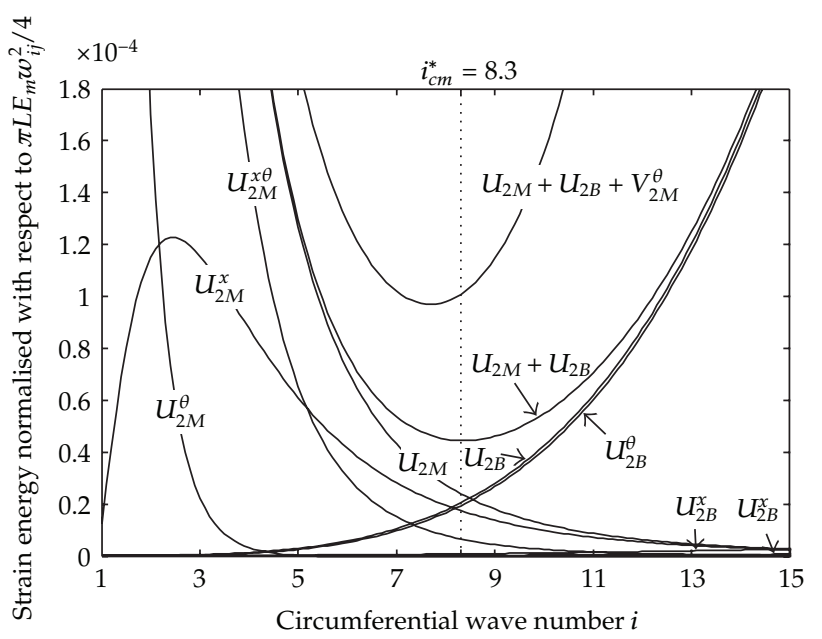

FIGURE 3: Energy contributions in the classical critical mode $j=1$ for the shell $\left[0^{\circ}\right]_{6}$.

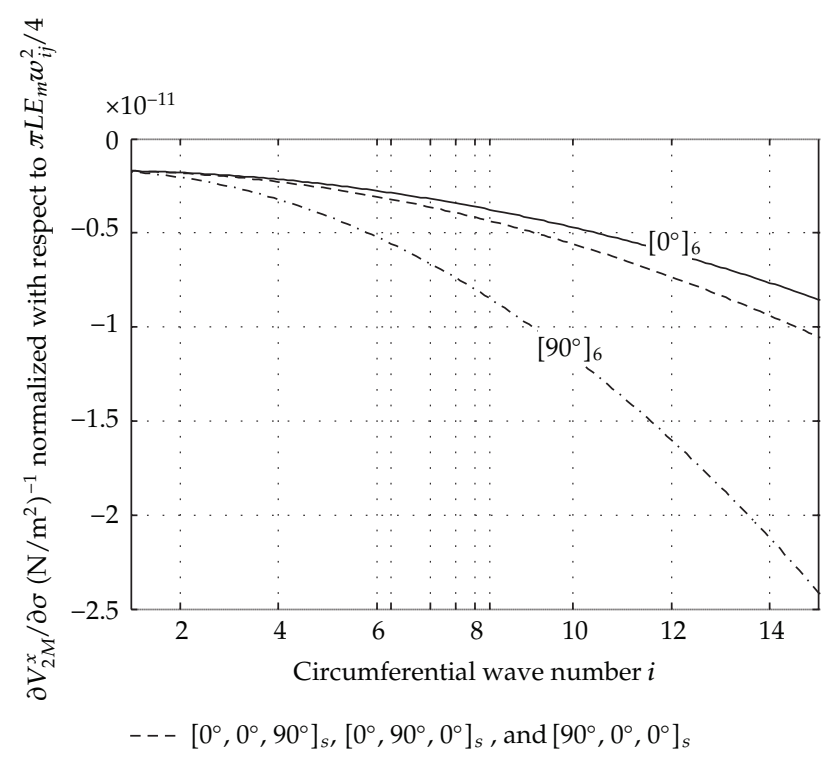

FIgURE 4: $\partial V_{2 M}^{x} / \partial \sigma$ of each case in the classical critical mode $j=1$.

membrane stiffness accompanying a reorientation of fibres into the circumferential direction has two counter vailing effects. The increase in circumferential bending energy will enhance while the increase wavelength of the critical buckling mode will reduce the buckling resistance. The optimum will represent a compromise of these two effects. But there is a further complicating factor. The decrease of the axial membrane stiffness will increase the destabilising effect of the nonlinear axial membrane energy as seen in Figure 4. The effect is an increase in the denominator of (21), resulting in a reduction of the reduced stiffness critical load. Therefore there must be an optimum choice of redistributing fibres in the axial and hoop direction to achieve the highest reduced stiffness critical load.

The analysis results of an extreme case $\left[90^{\circ}\right]_{6}$, that is moving all fibres from the axial direction to the hoop direction, are shown in Table 1. As aforementioned, the excessive compromise of the axial membrane stiffness caused a significant circumferential mode shift from 8.3 to 6.3. The relatively large increase of the circumferential wave length counteracted the effort to make a substantial enhancement of the hoop bending energy through moving all fibres from the axial direction to the hoop direction. The hoop bending energy and the total bending energy show a slight increase, whereas the decreased axial membrane stiffness substantially increased the destabilising effect of the nonlinear axial membrane energy; see Figure 4. The overall effect caused a nearly $30 \%$ reduction of the reduced stiffness critical load. It is clear that an optimum reduced stiffness critical load should have a laminate configuration with fibres distributed in both directions.

Moving only part of the axial fibres to the hoop direction, say $\left[0^{\circ}, 0^{\circ}, 90^{\circ}\right]_{s}$, the angles of fibre orientation are counted from the outside to the middle plane of the shell and "s" represents symmetric distribution about the middle plane. From Table 1, it can be seen that the linear hoop bending energy and the total bending energy had a slight reduction due to the moderate mode shift from 8.3 to 8 . The destabilising effect of the nonlinear axial membrane energy was also moderately increased. But the overall effect resulted in a slightly higher reduced stiffness critical load compared with the case of $\left[90^{\circ}\right]_{6}$. In addition to the mode shift, the other reason why the linear hoop bending energy was not increased substantially is that the hoop plies were placed near the middle plane of the shell and hence have a small eccentricity. To more effectively enhance the linear hoop bending energy, the hoop fibres should be moved from the middle plane to the surface of the shell to increase the eccentricity. This can also be justified with the interpretation of energy contributions. Since restacking the lamina sequence will not change the membrane stiffness, moving hoop fibres from the middle plane to the surface will definitely lead to the net increase of the hoop bending energy, see Figure 2, and the decrease of the linear axial bending energy will have negligible effect [11]. With the increase of the linear hoop bending energy, the classical critical mode will have a moderate shift to increase the circumferential wave length, having the effect of decreasing the destabilising effect of the nonlinear axial membrane energy; see Figure 4. The overall effect will definitely lead to a net increase of the reduced stiffness critical load. The cases $\left[0^{\circ}, 0^{\circ}, 90^{\circ}\right]_{s}$, $\left[0^{\circ}, 90^{\circ}, 0^{\circ}\right]_{s}$, and $\left[90^{\circ}, 0^{\circ}, 0^{\circ}\right]_{s}$ in Table 1 illustrate the procedure. With the moderate mode shifts, the linear hoop bending energy, the total bending energy, and the reduced stiffness critical load were all increased significantly. The last two cases in Table 1 illustrate a continuation of the idea of moving axial fibres to the hoop direction. However, again excessive sacrifice of the axial membrane stiffness combined with relatively small eccentricity of the hoop plies leads to a net decrease of the reduced stiffness critical load compared with the case $\left[90^{\circ}, 0^{\circ}, 0^{\circ}\right]_{s}$.

3.2. Anisotropic Laminate Configurations . The effect of changing the angle of fibre orientation is equivalent to changing the membrane and bending stiffnesses. Hence, the reduced stiffness optimisation can still be carried out within 
TABLE 1: Reduced stiffness analysis results for each orthotropic shell.

\begin{tabular}{|c|c|c|c|c|}
\hline Orthotropic laminate configuration & $\begin{array}{c}\sigma_{c m}^{*}\left(i_{c m}^{*}, j_{c m}^{*}\right) \\
\left(\times 10^{7} \mathrm{~Pa}\right)\end{array}$ & $\begin{array}{c}U_{2 B} \\
\left(\times 10^{-5}\right) \\
\end{array}$ & $\begin{array}{c}U_{2 B}^{\theta} \\
\left(\times 10^{-5}\right)\end{array}$ & $\begin{array}{c}\partial V_{2 M}^{x} / \partial \sigma_{c m}^{*} \\
\left(\times 10^{-12}\right)\left(\mathrm{N} / \mathrm{m}^{2}\right)^{-1}\end{array}$ \\
\hline$\left[0^{\circ}\right]_{6}$ & $0.56(8.3,1)$ & 2.139 & 2.030 & -3.811 \\
\hline$\left[90^{\circ}\right]_{6}$ & $0.40(6.3,1)$ & 2.256 & 2.195 & -5.697 \\
\hline$\left[0^{\circ}, 0^{\circ}, 90^{\circ}\right]_{s}$ & $0.47(8.0,1)$ & 2.001 & 1.899 & -4.234 \\
\hline$\left[0^{\circ}, 90^{\circ}, 0^{\circ}\right]_{s}$ & $0.59(7.6,1)$ & 2.354 & 2.262 & -3.984 \\
\hline$\left[90^{\circ}, 0^{\circ}, 0^{\circ}\right]_{s}$ & $0.78(7.1,1)$ & 2.890 & 2.812 & -3.690 \\
\hline$\left[90^{\circ}, 0^{\circ}, 90^{\circ}\right]_{s}$ & $0.58(6.8,1)$ & 2.515 & 2.443 & -4.321 \\
\hline$\left[90^{\circ}, 90^{\circ}, 0^{\circ}\right]_{s}$ & $0.66(6.7,1)$ & 2.803 & 2.734 & -4.243 \\
\hline
\end{tabular}

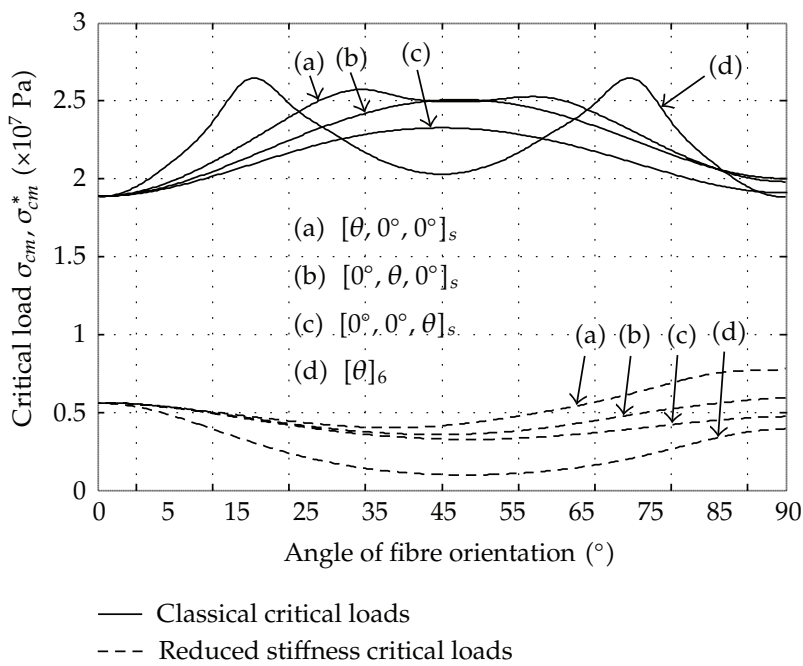

Figure 5: Classical critical loads and reduced stiffness critical loads versus the varying angle of fibre orientation of a series of anisotropic cases.

the framework of energy contributions. Figure 5 shows a reduced stiffness optimisation including the effect of any angle of fibre orientation. Firstly, the case $[\theta]_{6}$ in which fibres are unidirectionally oriented in any direction was taken as a start point. From Figure 5, it can be seen that $\left[0^{\circ}\right]_{6}$ gives the highest reduced stiffness critical load. Based on the case $\left[0^{\circ}\right]_{6}$ and under the consideration of improving the linear hoop bending energy, the angle of orientation of a part of fibres was taken as design variable to examine its effect on the reduced stiffness critical load. From Figure 5, it is clear that the orthotropic case $\left[90^{\circ}, 0^{\circ}, 0^{\circ}\right]_{s}$ still gives the highest reduced stiffness critical load. Based on the case $\left[90^{\circ}, 0^{\circ}, 0^{\circ}\right]_{s}$, the same approach was applied. From Figure 6 it can be observed that the case $\left[90^{\circ}, 0^{\circ}, 0^{\circ}\right]_{s}$ still stands out as providing the optimum solution.

Meanwhile, it is worth noticing from Figures 5 and 6 that laminate configurations with high classical critical loads such as $\left[20^{\circ}\right]_{6},\left[70^{\circ}\right]_{6},\left[0^{\circ}, 0^{\circ}, 45^{\circ}\right]_{s},\left[0^{\circ}, 45^{\circ}, 0^{\circ}\right]_{s},\left[35^{\circ}, 0^{\circ}\right.$, $\left.0^{\circ}\right]_{s},\left[55^{\circ}, 0^{\circ}, 0^{\circ}\right]_{s},\left[90^{\circ}, 0^{\circ}, 45^{\circ}\right]_{s}$, and $\left[90^{\circ}, 45^{\circ}, 0^{\circ}\right]_{s}$ are always associated with relatively low lower-bound buckling loads. This indicates the high imperfection sensitivity of these laminate configurations and emphasises the errors in common attempts to optimise the upper-bound classical

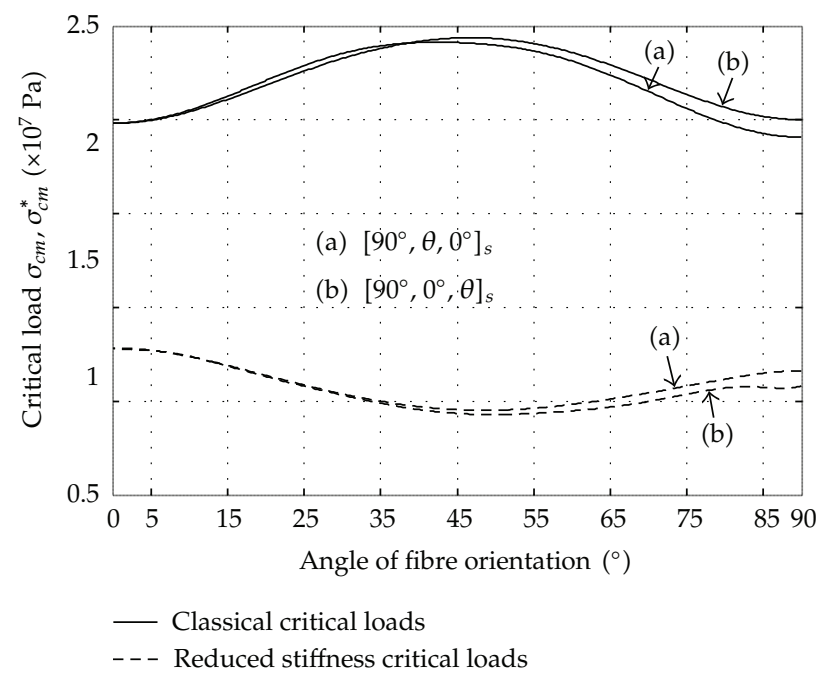

FIGURE 6: Classical critical loads and reduced stiffness critical loads versus the varying angle of fibre orientation of the case $\left[90^{\circ}, 0^{\circ}, \theta\right]_{s}$ and $\left[90^{\circ}, \theta, 0^{\circ}\right]_{s}$.

critical loads. It is apparent that the presence of initial imperfections may render the predicted optimal configurations for perfect shells unfavorable compared with configurations exhibiting lower imperfection sensitivity of buckling load carrying capacities.

3.3. Effect of Fibre Volume Fraction. So far the case $\left[90^{\circ}, 0^{\circ}\right.$, $\left.0^{\circ}\right]_{s}$ with the fibre volume fraction of each ply fixed at 0.5 gives the highest reduced stiffness critical load. To further optimise energy contributions so as to enhance the reduced stiffness critical load, we can take the fibre volume fraction of the hoop ply as an additional design parameter. Figure 7 shows the effect of increasing the fibre volume fraction in the outer lamina; 0.75 is assumed to be the fibre packing limit. In each case the volume fractions in each of the inner laminae are equal and adjusted to ensure that the total fibre volume fraction is fixed at 0.5. All of these results can be interpreted within the framework of the energy contributions. From Figure 7, the highest reduced stiffness critical load occurs at 0.75 and has the value of $9.1 \mathrm{MPa}$ which is $62.5 \%$ higher than $5.6 \mathrm{MPa}$ of the starting case $\left[0^{\circ}\right]_{6}$ and over $900 \%$ higher than the lowest load so far obtained for the case $\left[50^{\circ}\right]_{6}$ (see Figure 5). 


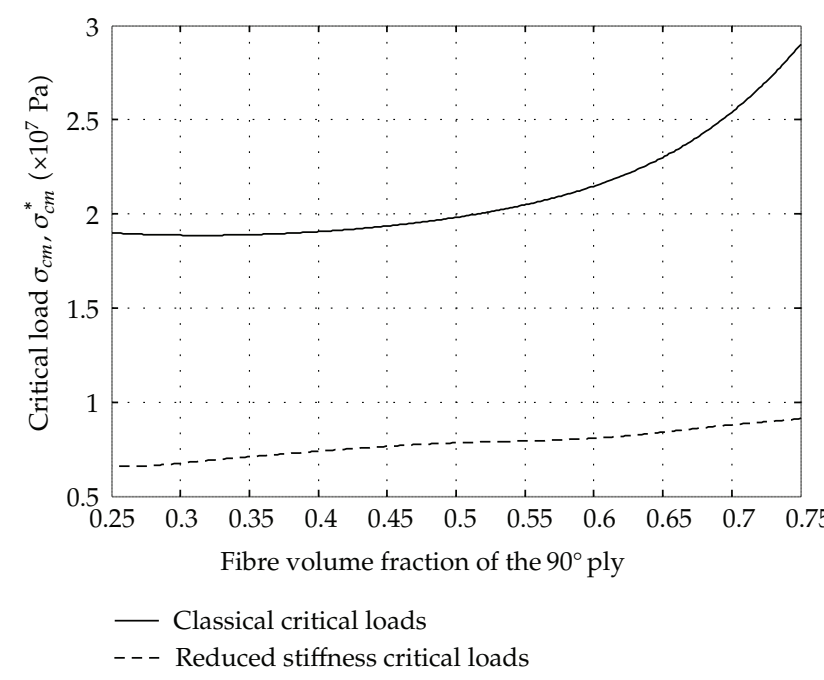

FIgURe 7: Classical critical loads and reduced stiffness critical loads versus the varying fibre volume fraction in the hoop direction in the case $\left[90^{\circ}, 0^{\circ}, 0^{\circ}\right]_{s}$ with the total fibre volume fraction fixed at 0.5 .

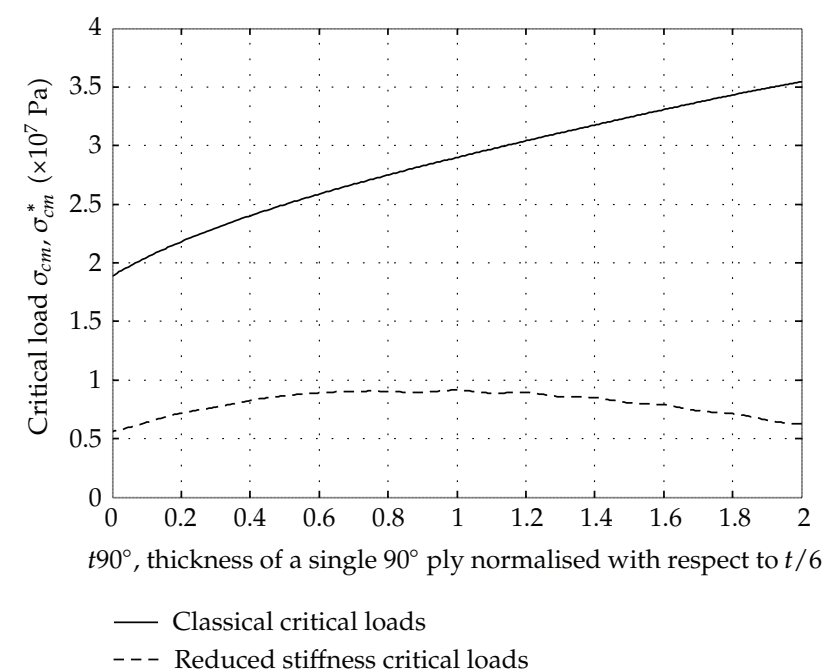

FIGURE 8: Classical critical loads and reduced stiffness critical loads versus the varying thickness of a single $90^{\circ}$ ply in the case of $\left[\left(90^{\circ}\right)_{t 90^{\circ}},\left(0^{\circ}\right)_{t 0^{\circ}},\left(0^{\circ}\right)_{t 0^{\circ}}\right]_{s}$ with the total shell thickness unchanged, the total fibre volume fraction fixed at 0.5 , and the fibre volume fraction of the $90^{\circ}$ ply fixed at 0.75 .

3.4. Effect of Lamina Thickness. Based on the above obtained laminate configuration we can further try to enhance the reduced stiffness critical load by changing the thickness of each hoop ply. Figure 8 shows such an analysis with the total thickness of the shell unchanged, the fibre volume fraction of the hoop ply kept constant at 0.75 and the fibre volume fractions in the axial plies adjusted to maintain a total fibre volume fraction of 0.5. It can be seen that the laminate configuration giving the highest reduced stiffness critical load is that for which all laminae have equal thickness. The change in any lamina thickness will break the balance of the so far optimised energy contributions and

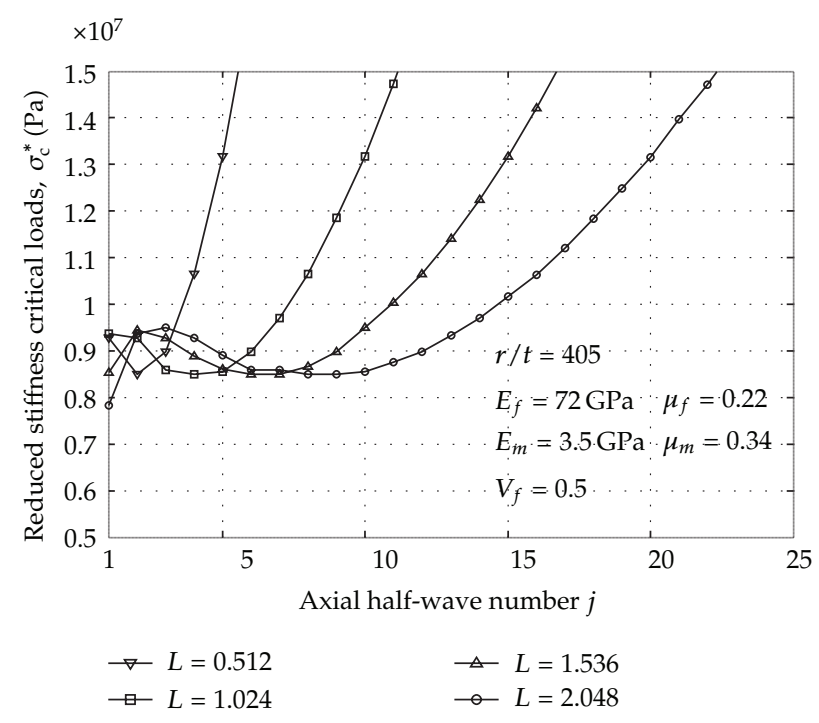

FIGURE 9: Effect of length to radius ratios on the reduced stiffness critical load spectra for the case of $\left[90^{\circ}, 0^{\circ}, 0^{\circ}\right]_{\text {s }}$ with $R=405$.

hence lead to the reduction in the reduced stiffness critical load.

3.5. Effect of Geometric Parameters. For the aforementioned illustrated case, the least values of the reduced stiffness critical loads for all laminate configurations occurred in the mode of $j=1$. Figure 9 shows the reduced stiffness critical loads with different length to radius ratios for the case $\left[90^{\circ}, 0^{\circ}, 0^{\circ}\right]_{s}$. It can be seen that the least values of the reduced stiffness critical loads can happen in modes other than $j=1$. For example, for $L=0.512$, the least reduced stiffness critical load occurs in mode $j=2$; for $L=1.024$, the least load happens in mode $j=4$; and for $L=1.536$, the least value occurs in mode $j=7$.

For cases where the least reduced stiffness critical loads occur in modes other than $j=1$, the energy components in each of the possible buckling modes have different contributions to shell's initial buckling resistance, and their respective importance in the corresponding buckling modes needs to be reexamined. Figures $10(\mathrm{a}), 10(\mathrm{~b})$, and $10(\mathrm{c})$ show the energy contributions in different $j$ modes for the case of $\left[90^{\circ}, 0^{\circ}, 0^{\circ}\right]_{S}$ with $L=1.024$ and $R=405$. As shown in Figure 10(a), in mode $j=1$, the nonlinear circumferential energy contributes nearly a third to the shell's initial buckling resistance; the linear circumferential bending energy accounts for the major part of the total bending energy; the linear axial membrane energy and the linear shear membrane energy have nearly the equal weights in constituting the total linear membrane energy; the contributions of the linear axial bending, the linear twist bending, and the linear circumferential membrane energies can be negligible. While in mode $j=4$, see Figure 10(b), the contribution of the nonlinear circumferential energy is far less than in mode $j=1$, the linear circumferential bending energy still takes a major role in the constitution of the total bending energy with the importance of the linear axial bending and the linear 


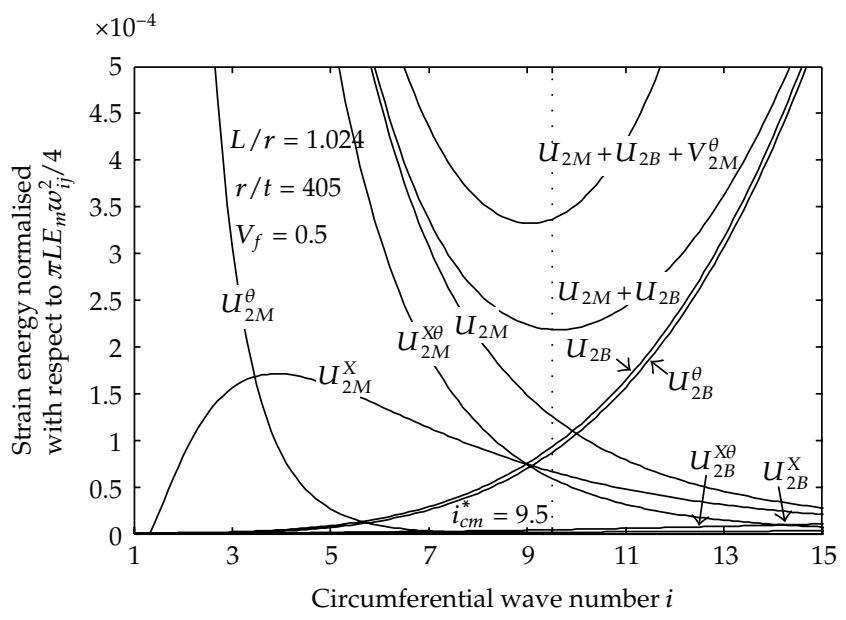

(a)

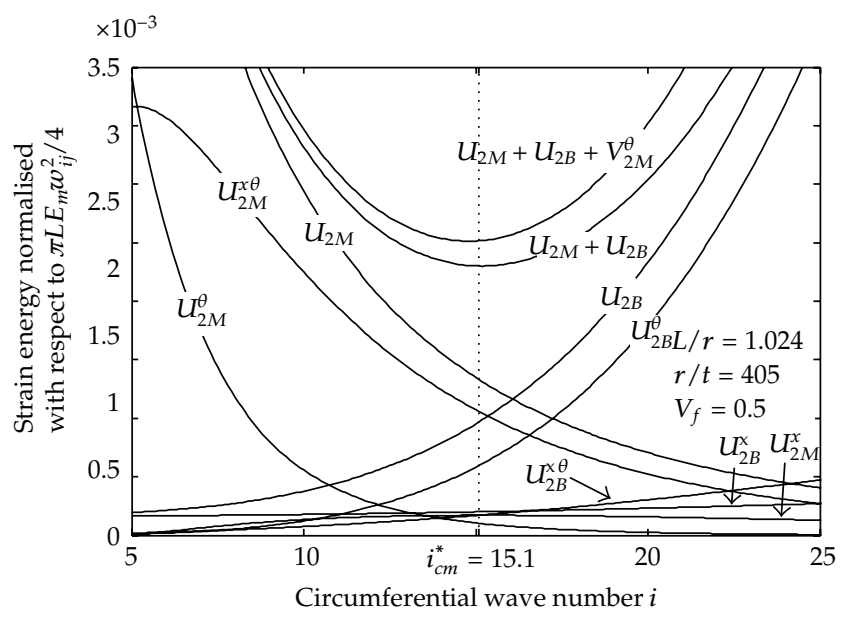

(b)

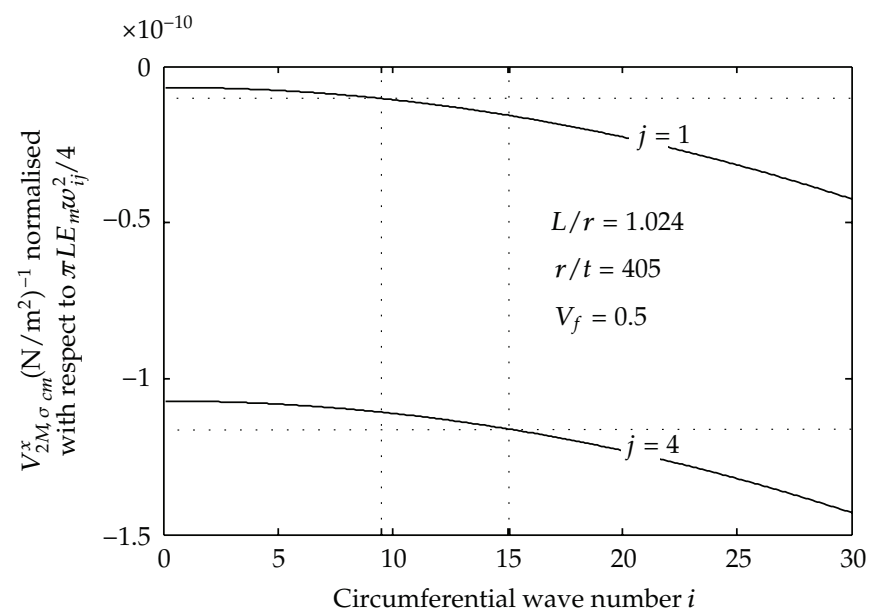

(c)

Figure 10: Energy contributions in the classical critical mode $j=1$ and $j=4$ for the case of $\left[90^{\circ}, 0^{\circ}, 0^{\circ}\right]_{\mathrm{s}}$ with $R=405$ and $L=1.024:(\mathrm{a})$ in mode $j=1$, (b) in mode $j=4$, and (c) nonlinear axial membrane energy component in $j=1$ and $j=4$.

twist bending energies increased a lot; for the total linear membrane energy, the importance of the linear shear membrane energy rises significantly compared to the dramatic reduction of the linear circumferential membrane energy due to the shortened circumferential wave length. Figure 10(c) shows the difference of the destabilising nonlinear axial membrane term in mode $j=1$ and $j=4$. For this case, the overall effect causes the least reduced stiffness critical load occur in mode $j=4$. Accordingly, the lower-bound reduced stiffness optimisation should look at the importance of those energy components in each of the possible buckling modes.

Geometric parameter studies for the case of $\left[90^{\circ}, 0^{\circ}, 0^{\circ}\right]_{s}$ are carried out in Figures 11 and 12 to examine the effects of length to radius ratio and radius to thickness ratio on the classical critical loads and reduced stiffness critical loads. As shown in Figure 11, for moderately long shells, the minimum classical critical loads and minimum reduced stiffness critical loads are somewhat independent of the length to radius ratio. In Figure 12, there is a monotonic decrease in both the minimum classical critical loads and minimum reduced stiffness critical loads with increasing radius to thickness ratio.

\section{Conclusions}

The reduced stiffness method has been extended to the lower-bound buckling load analysis of FPR-laminated shells. With the understanding derived from analysis of the different contributions of various energy components to the buckling resistance of the shell, a physically based optimisation was carried out with respect to the safe lower bounds provided by a reduced stiffness critical load. To illustrate the approach, an example of a simply supported, 6-ply, symmetric glassepoxy circular cylindrical shell under uniform axial load is considered. By choosing appropriate combinations of the many geometric and material design variables, the lowerbound buckling load is shown to be enhanced significantly. Increasing the hoop bending stiffness has considerable 


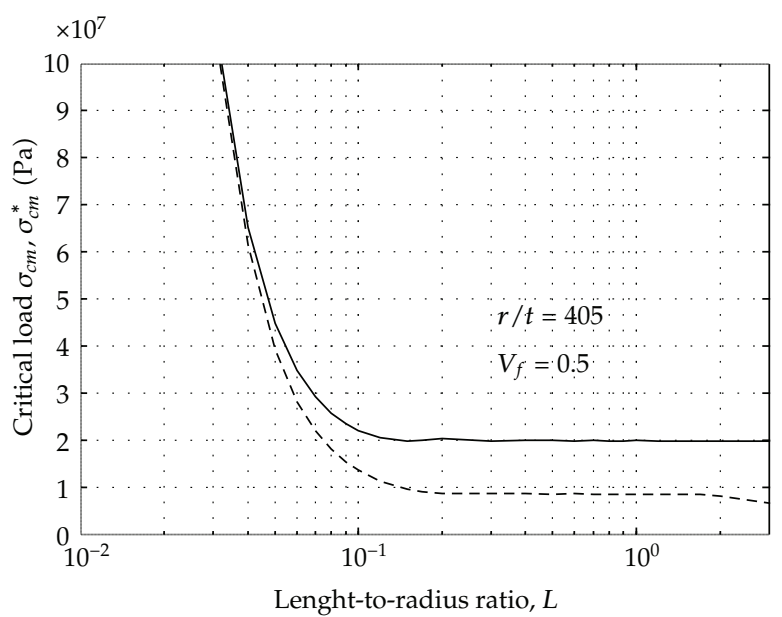

— Minimum classical critical loads

- - - Minimum reduced stiffness critical loads

FIGURE 11: Effect of length to radius ratios on the reduced stiffness critical load spectra for the case of $\left[90^{\circ}, 0^{\circ}, 0^{\circ}\right]_{\mathrm{s}}$ with $R=405$.

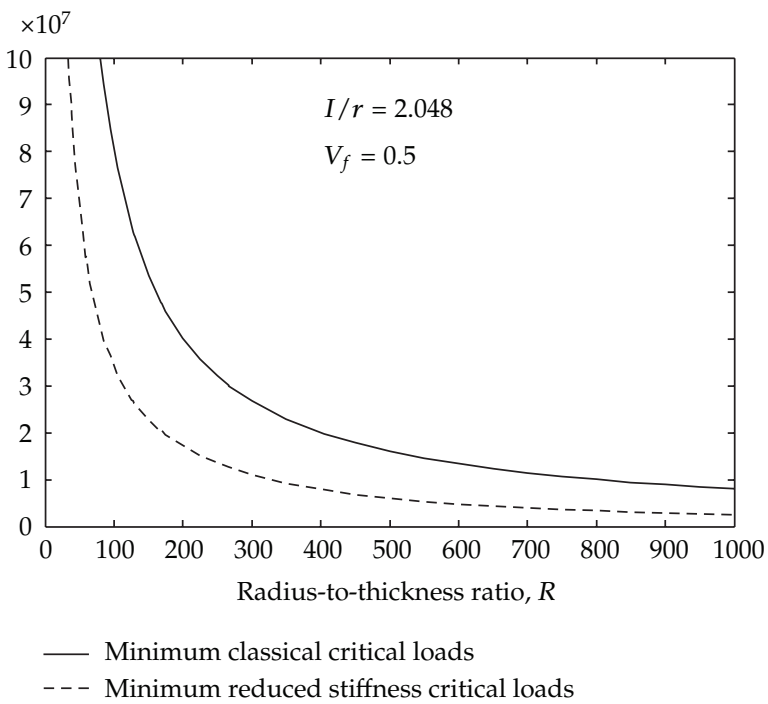

FIGURE 12: Effect of radius to thickness ratio on the classical and reduced stiffness critical loads for the case of $\left[90^{\circ}, 0^{\circ}, 0^{\circ}\right]_{\mathrm{S}}$ with $L=$ 2.048 .

benefit in enhancing the lower-bound buckling load provided that the compromise of the axial membrane stiffness does not exceed certain limits. Shells with orthotropic laminate configurations exhibit more favorable lower-bound buckling resistance than anisotropic ones. High classical critical loads are often associated with relatively low lowerbound reduced stiffness critical loads, which indicate the high imperfection sensitivity. This seriously undermines the common attempts to optimise buckling loads of FRPlaminated composite shells on the basis of upper-bound classical critical loads.

Based on the sound physical argument, the RSM provides a framework to compute the lower-bound buckling loads from a linear eigenvalue analysis and encourages the delineation of those components of the shell's membrane and bending stiffness that are important and those that are unimportant within each of the prospective buckling modes. The simplicity of this analytically based method enables the prediction of and also gives a better interpretation of the likely consequences of variations of the many material and geometric parameters that govern the safe resistance to buckling. As a tool for guiding appropriate combinations of parameters to affect enhanced, or even "optimum", buckling capacities this approach has been shown to have considerable advantage over many of the currently available alternative means for improving design performance.

\section{References}

[1] Y. S. Nshanian and M. Pappas, "Optimal laminated composite shells for buckling and vibration," AIAA journal, vol. 21, no. 3, pp. 430-437, 1983.

[2] J. Onoda, "Optimal laminate configuration of cylindrical shells for axial buckling," AIAA journal, vol. 23, no. 7, pp. 1093-1098, 1985.

[3] G. Sun and J. S. Hansen, "Optimal design of laminated composite circular-cylindrical shells subject to combined loads," Journal of Applied Mechanics, Transactions ASME, vol. 55, no. 1, pp. 136-142, 1988.

[4] H. Fukunaga and G. N. Vanderplaats, "Stiffness optimization of orthotropic laminated composites using lamination parameters," AIAA journal, vol. 29, no. 4, pp. 641-646, 1991.

[5] C. G. Diaconu and H. Sekine, "Layup optimization for buckling of laminated composite shells with restricted layer angles," AIAA Journal, vol. 42, no. 10, pp. 2153-2163, 2004.

[6] R. C. Tennyson and J. S. Hansen, "Optimum design for buckling of laminated cylinders," in IUTAM Symp. on Collapse: The Buckling of Structures in Theory and Practice, J. M.T. Thompson and G. W. Hunt, Eds., pp. 409-429, Cambridge University Press, Cambridge, UK, 1982.

[7] M. D. Pandey and A. N. Sherbourne, "Imperfection sensitivity of optimized, laminated composite shells: a physical approach," International Journal of Solids and Structures, vol. 27, no. 12, pp. 1575-1595, 1991.

[8] J. G. A. Croll, "Towards a rationally based elastic-plastic shell buckling design methodology," Thin-Walled Structures, vol. 23, no. 1-4, pp. 67-84, 1995.

[9] R. M. Jones, Mechanics of Composite Materials, Taylor \& Francis, 2nd edition, 1999.

[10] S. Yamada, N. Yamamoto, J. G. A. Croll, and P. Bounkhong, "Local buckling criteria of thin-walled FRP circular cylinders under compression," in Proceedings of the International Colloquium on Application of FRP to Bridges, (ICAFB'06), Tokyo, Japan, 2006.

[11] H. Wang and J. G. A. Croll, "Optimising buckling capacities for composite shells," in Proceedings of the 3rd European Conference on Computational Mechanics-Solids, Structures and Coupled Problems in Engineering, Lisbon, Portugal, 2006. 

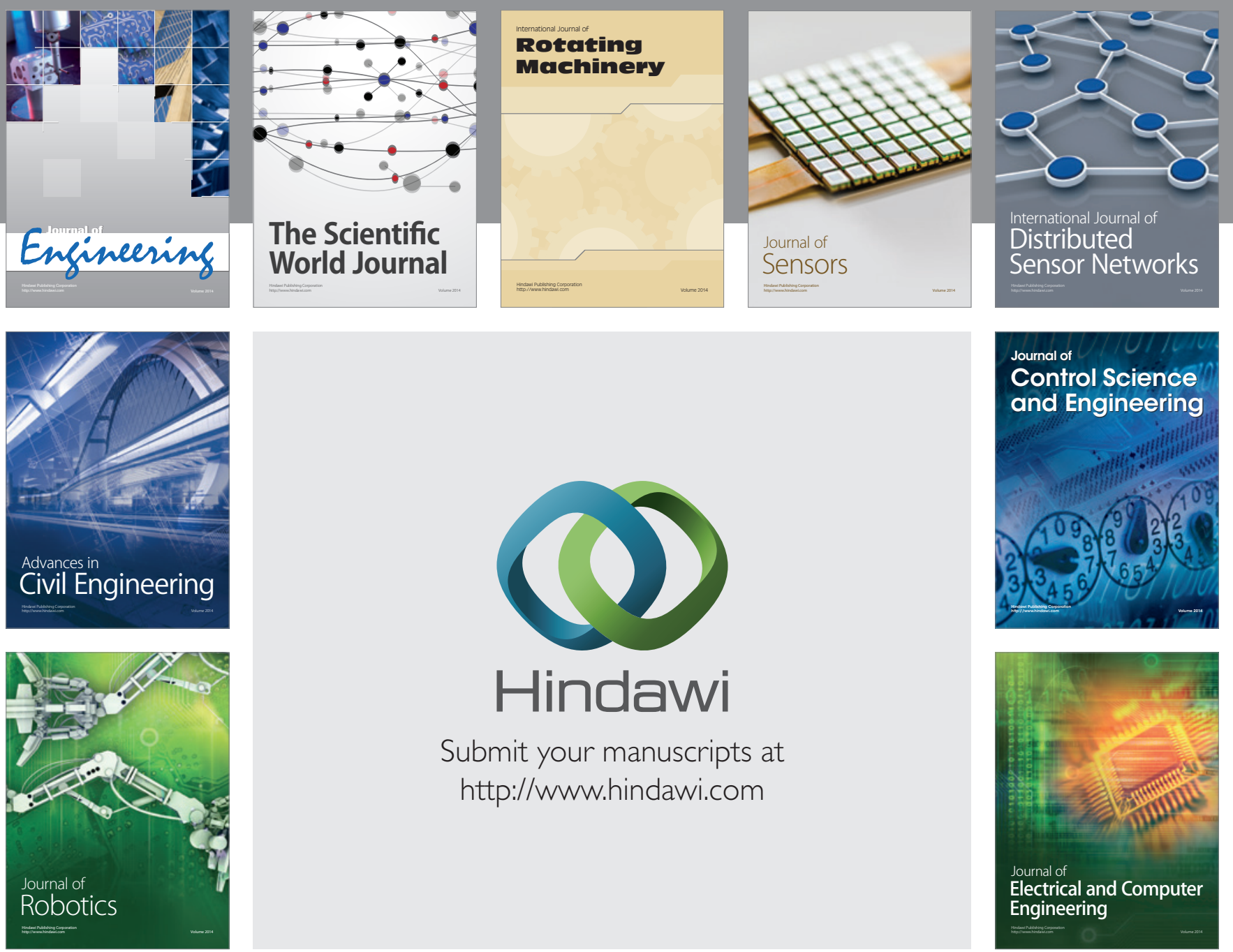

Submit your manuscripts at

http://www.hindawi.com
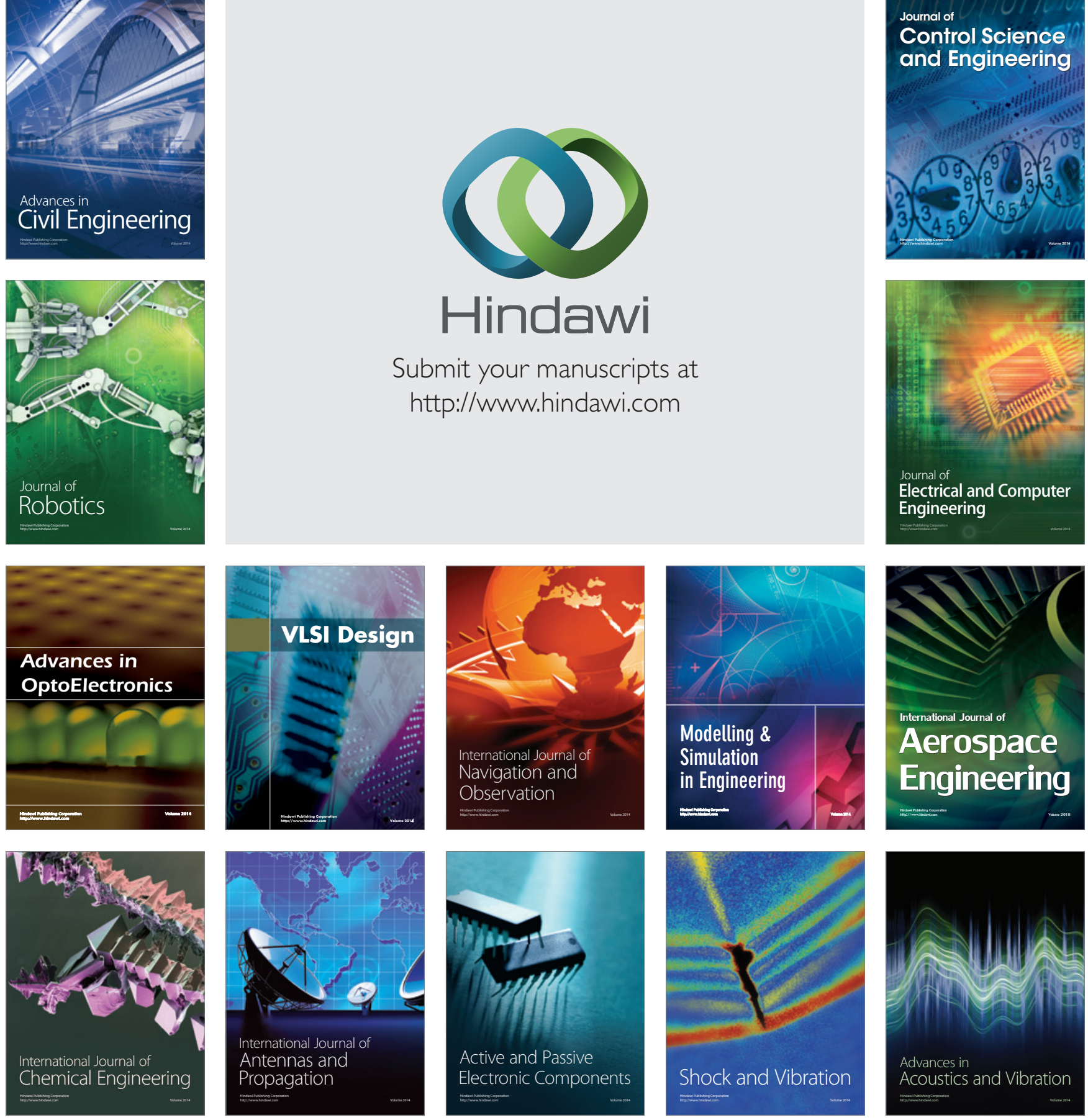